\title{
Asymptomatic rupture of cornual gestation presented as abdominal mass
}

\author{
Sabita Saichandran $^{1,2 *}$, Sunita Samal ${ }^{1}$ \\ ${ }^{1}$ Department of Obstetrics \& Gynecology, Mahatma Gandhi Medical College \& Research Institute, Pillayarkuppam, \\ Pondicherry-607402, India \\ ${ }^{2}$ Presently affiliated to Department of Obstetrics \& Gynecology, Indira Gandhi Medical College and Research \\ Institute, Pondicherry, India
}

Received: 30 September 2013

Accepted: 8 October 2013

*Correspondence:

Dr. Sabita Saichandran,

E-mail: sabita.kutty@gmail.com

(c) 2013 Saichandran S et al. This is an open-access article distributed under the terms of the Creative Commons Attribution Non-Commercial License, which permits unrestricted non-commercial use, distribution, and reproduction in any medium, provided the original work is properly cited.

\section{ABSTRACT}

We report a case of mass abdomen secondary to asymptomatic rupture of cornual pregnancy probably occurred 1-2 months prior to her visit. Uterus sparing surgery was performed following which she had spontaneous conception in less than a year with successful outcome.

Keywords: Cornual pregnancy, Asymptomatic rupture, Cornual repair, Future pregnancy outcome

\section{INTRODUCTION}

A cornual pregnancy refers to the implantation and development of a pregnancy in the lateral upper portion of the uterus, whereas an interstitial pregnancy implants within the myometrium of the proximal and intramural portion of the fallopian tube. ${ }^{1}$ Both the entities account for $2-4 \%$ of ectopic pregnancies and they tend to advance further than tubal and cervical pregnancies. When cornual pregnancy ruptures, there is disproportionately high incidence of hemoperitoneum and shock. Cornual pregnancy is traditionally treated surgically by either hysterectomy or cornual resection with simple suturerepair. This classical treatment of cornual pregnancies has an impact on future pregnancies in the form of recurrence and rupture of uterus in early gestation.

The aim of this paper is to report a case of asymptomatic rupture of cornual pregnancy with extrusion of fetus into the peritoneal cavity presenting as abdominal mass after few months. Following cornual repair she conceived spontaneously in a year with successful outcome.

\section{CASE REPORT}

A twenty three year old lady attended with history of abdominal mass with pain and amenorrhoea for 6 months. She was diagnosed as a case of adnexal mass by a local practitioner. Her previous menstrual cycles were regular. She gave birth to a healthy baby by vaginal delivery three years ago. There was no history of insertion of IUCD. On clinical examination she was anemic; no other abnormality was detected. On abdominal examination a mass corresponding to a size of 20 weeks of pregnant uterus with variable consistency was found. It was tender on deep palpation and had a smooth surface. On percussion no free fluid was found in the abdomen. On speculum examination, cervix was found to be congested and vaginal examination revealed mobile and non tender uterus of normal size. The mass was not palpable through the fornix. Based on these findings a provisional diagnosis of ovarian tumor was made. All routine investigations and serum beta HCG were reported to be within normal limits except for mild anemia. Trans-abdominal sonography revealed a large 
solid cystic mass measuring 9.2 X 11.6 X $8.5 \mathrm{~cm}$. A nonviable fetus was seen in the left lateral aspect of the mass. The mass was found separate from uterus and fetus and was probably suggestive of abdominal ectopic pregnancy with fetal demise. No fetal skeleton was found in the abdominal X-ray.

Laparotomy was performed. The placenta with intact sac was found in the midline in the umbilical region, being adherent to the omentum and right cornual area and was connected to the fetus by a thin umbilical cord. Body of the fetus with legs was found in between the uterus and urinary bladder while the fetal head being located in the left iliac fossa adherent to the lateral pelvic wall (Figure 1, 2). Bilateral tubes and ovaries were healthy (Figure 3 ). All the contents were removed and cornual rent repair was done. The specimen was sent for histopathological examination which confirmed the presence of fetus and placental tissue. Post-operative period was uneventful and she was discharged on sixth post operative day after counseling on future pregnancy. During her follow-up visits she had minor complaints. After 14 months of her discharge from the hospital, she presented with history of amenorrhea for 3 months. Trans abdominal sonography revealed pregnancy of 15-16 weeks and evaluation was made with a particular reference to placental localization. The placenta was found to be fundal anterior and left. She was admitted during thirty second week with pain abdomen which subsided spontaneously. She was advised to stay in the hospital for safe confinement with a plan to undertake elective LSCS after the attainment of fetal maturity. During the thirty fourth week she developed abdominal pain. In view of pain and irritable uterus, emergency caesarean was done. A live born baby with weight of $2 \mathrm{~kg}$ was delivered. No neonatal complications occurred. The previous uterine scar was found to be intact and right and left tube were normal. Tubal ligation was done. There was no intraoperative and postoperative complication. She was discharged on seventh postoperative day. Her post partum period was uneventful.

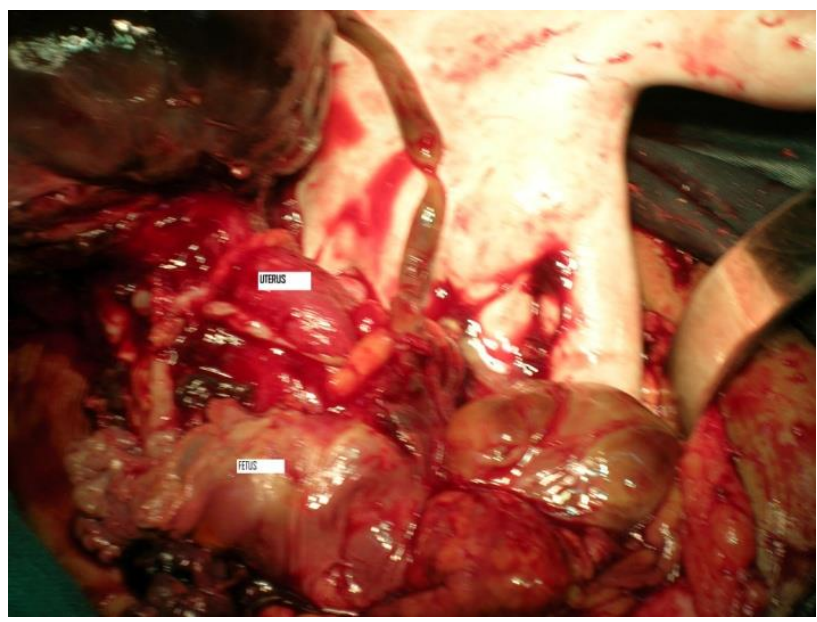

Figure 1: Showing fetus connected with umbilical cord.

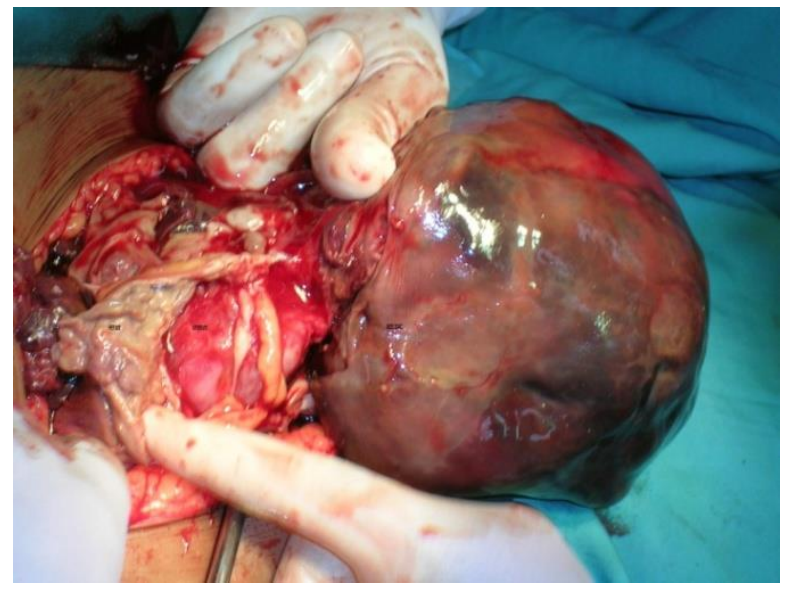

Figure 2: Showing fetus in uterovesical pouch.

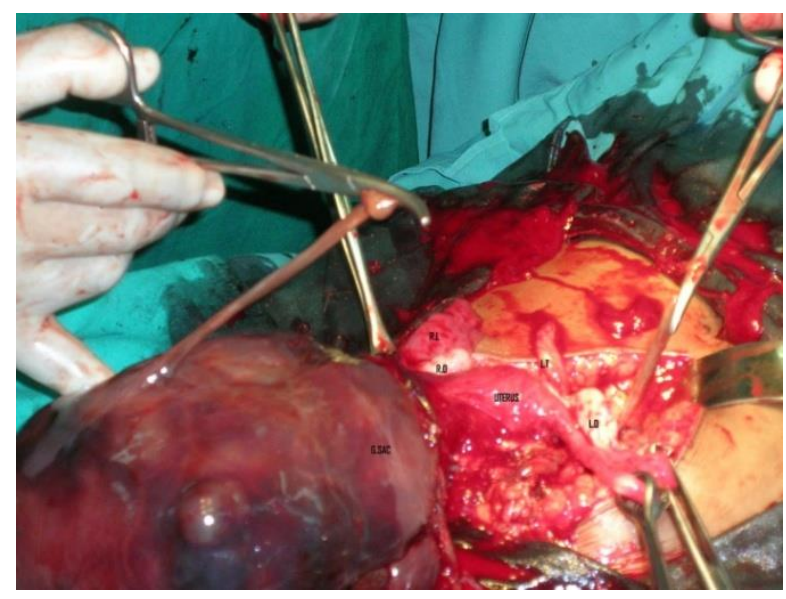

Figure 3: Uterus with bilateral intact tubes and ovaries and showing gestational sac attached to the right cornual region (clamp in the cornual region).

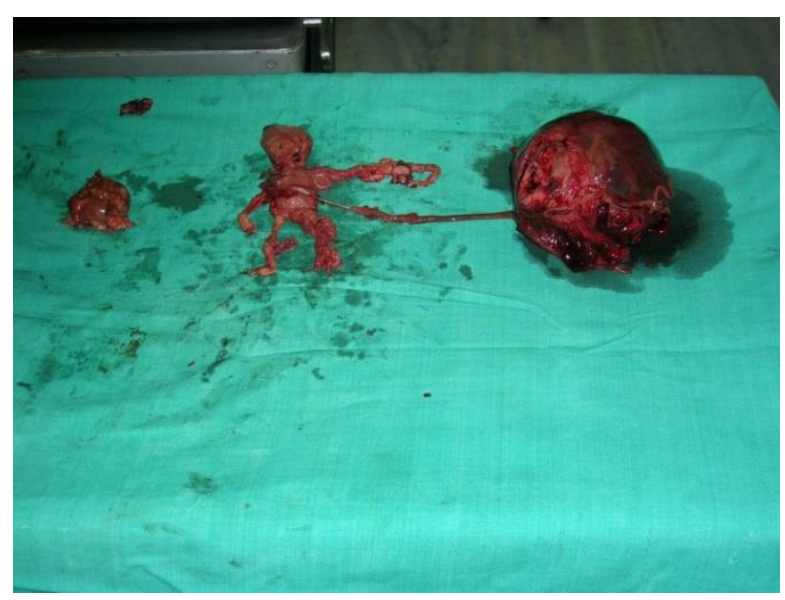

Figure 4: Total fetus.

\section{DISCUSSION}

Cornual pregnancy is a rare form of ectopic pregnancy. The etiologic factors for cornual pregnancy are pelvic inflammatory disease, tumor, Assisted Reproductive Techniques, ${ }^{2}$ Quality of the embryos and the hormonal 
milieu at the moment of embryo transfer, ${ }^{3}$ bilateral salpingectomy ${ }^{4}$ and peritubular and intratubular adhesions related or not related to endometriosis are the other additional risk factors. In our patient no other cause than PID could be implicated.

Cornual pregnancies often rupture later than other tubal pregnancies because the myometrium is more distensible than the fallopian tube. Twenty percent of cases that advance beyond 12 weeks of gestation ends in rupture and usually results in brisk hemorrhage and higher maternal morbidity and mortality rates than other ectopic pregnancies. Because of these late presentations uterine rupture is a potentially catastrophic event requiring laparotomy and hysterectomy leading to the premature end of reproductive capacity.

In contrast to the cases reported earlier, our patient had asymptomatic rupture, two months after which she presented as mass per abdomen. There is a report of cornual pregnancy persisting until fetal viability confirmed by ultrasound and diagnosed as a case of asymptomatic uterine rupture managed by abdominal delivery of live fetus. ${ }^{5}$ Since our patient was hemodynamically stable, we proceeded with cornual repair with a concern to save her reproductive capacity.

When an unruptured cornual pregnancy is diagnosed, various options for conservative management with uterine preservation have been described in literature such as parenteral methotrexate, ultrasound guided direct injection of methotrexate or potassium chloride into the cornual gestational sac, laparoscopic cornual resection and selective uterine artery embolization. ${ }^{6-8}$

Some authors have reported on performing cornual resection without suturing the myometrial defect. ${ }^{9}$ One of the major advantages of conservative treatment of cornual pregnancy is the preservation of fertility and the main disadvantages are the recurrence and risk of uterine rupture during the subsequent pregnancy. Counselling the patients for increasing the inter-pregnancy interval is important in reducing the maternal and fetal morbidity. In our case she conceived in less than 1 year and attended for antenatal evaluation at $14^{\text {th }}$ week of gestation and she had appropriate antenatal care and timely intervention resulting in successful pregnancy.

\section{REFERENCES}

1. Malinowski, Bates SK: Semantics and pitfalls in the diagnosis of corneal/interstitial pregnancy. Fertil Steril 2006;86:1764.

2. Chang Y, Lee JN, Yang CH, Hsu SC, Tsai EM. An unexpected quadruplet heterotopic pregnancy after bilateral salpingectomy and replacement of three embryos, Fertil Steril 2003;80:218-220.

3. Pan HS, Chuang J, Chiu SF, Hsieh BC, Lin YH, Tsai YL, Huang SC, Hsieh ML, Chen CY, Hwang JL. Heterotopic triplet pregnancy: report of a case with bilateral tubal pregnancy and an intrauterine pregnancy. Hum Reprod 2002;17:1363-1366.

4. Agarwal SK, Wisot AL, Garzo G, Meldrum DR. Cornual pregnancies in patients with prior salpingectomy undergoing in vitro fertilization and embryo transfer, Fertil Steril 1996;65:659-660.

5. Brewer H, Gefroh S, Munkarah A, Hawkins R, Redman ME. Asymptomatic uterine rupture of a cornual pregnancy in the third trimester: a case report. J Reprod Med 2005;50:715-718.

6. Valsky DV, Hamani Y, Verstandig A, Yagel S. The use of 3D rendering, VCI-C, 3D power Doppler and $\mathrm{B}$-flow in the evaluation of interstitial pregnancy with arteriovenous malformation treated by selective uterine artery embolization. Ultrasound Obstet Gynecol 2007;29:352-355.

7. Tulandi T, Vilos G, Gomel V. Laparoscopic treatment of interstitial pregnancy. Obstet Gynecol 1995;83:465-467.

8. Woodland MB, Depasquale SE, Molinari JA, Sagullo CC. Laparoscopic approach to interstitial pregnancy. J Am Assoc Gynecol Laparosc 1995;3: 439-441.

9. Gezer A, Mutlu H. Laparoscopic management of cornual pregnancy without sutures Arch Gynecol Obstet 2004;270:194-196.

DOI: $10.5455 / 2320-1770 . i j \operatorname{cog} 20131261$

Cite this article as: Saichandran S, Samal S.

Asymptomatic rupture of cornual gestation presented as abdominal mass. Int J Reprod Contracept Obstet Gynecol 2013;2:746-8. 